Please do not remove this page

RMIT

UNIVERSITY

\title{
Adaptive LMS ternary filtering
}

Sadik, Amin; Hussain, Zahir

https://researchrepository.rmit.edu.au/esploro/outputs/9921861929201341/filesAndLinks?institution=61RMIT_INST\&index=null

Sadik, A., \& Hussain, Z. (2005). Adaptive LMS ternary filtering. Proceedings of the TENCON 2005 IEEE Region 10 Conference, 1-3. https://doi.org/10.1109/TENCON.2005.301285

Published Version: https://doi.org/10.1109/TENCON.2005.301285

Repository homepage: https://researchrepository.rmit.edu.au

(c) 2005 IEEE. Personal use of this material is permitted. However, permission to reprint/republish this material for advertising or promotional purposes or for creating new collective works for resale or redistribution to servers or lists, or to reuse any copyrighted component of this work in other works must be obtained from the IEEE.

Downloaded On 2023/04/26 21:49:33 +1000 


\section{Adaptive LMS Ternary Filtering}

\author{
Amin Z. Sadik \\ School of Engineering Systems \\ Queensland University of Technology \\ (Currently a Visiting Researcher at SECE \\ RMIT, Melbourne, Australia) \\ E-mail: amin.sadik@rmit.edu.au
}

\author{
Zahir M. Hussain, SMIEEE \\ School of Electrical \& Computer Engineering \\ RMIT, Melbourne, Australia \\ Email: zmhussain@ieee.org
}

\begin{abstract}
The unresolved problem of finding an adaptive LMS structure for ternary filters has substantially limited their useability despite their major advantage of hardware simplicity. In this paper we tackle this challenging problem by introducing an algorithm that seems to be quite promising. Results were surprising as it appeared that the proposed ternary algorithm is comparable in performance to the multi-bit Wiener LMS algorithm. we expect that this approach will open the door for ternary systems to be ready for replacing multi-bit signal processing systems.
\end{abstract}

\section{INTRODUCTION}

Ternary filtering is a new digital signal processing (DSP) approach that has introduced substantial hardware advantage over multi-bit systems [1]. The ternary filter is an FIR filter with ternary taps (i.e., $+1,0,-1$ ); it is most efficient when the input signal is in single-bit format [2]. Several recent works have made the theory of ternary filtering nearly mature and ready for application [3], [4], [5], [6], [7], [8], [9]. However, for a new filtering theory to be of large scale application as a substitute for the traditional multi-bit DSP systems, an efficient adaptive structures are inevitable. This is so as most applications are challenged by noise and time-varying conditions. In fact, one of the major drawbacks that hindered the analog signal processing (ASP) for decades was the lack of adaptivity. These days the demand for adaptive filtering can be found in nearly all applications, especially in communication systems.

Unfortunately, there is no adaptive LMS structure of any kind for ternary filtering. The challenge of this problem is the harsh quantization that prevents straightforward LMS application. In this paper we attempt to solve this problem by proposing an efficient structure for adaptive noise reduction. This application is of major significance in many applications, such as communication channel equalization. The results were quite astonishing as the performance of this simple structure is comparable to that of multi-bit LMS Wiener algorithm.

This structure can be extended to a general adaptive structure. We are currently continuing research in this direction to finalize its theory.

\section{Structure of Non-Adaptive Ternary Filters}

The ternary filter is a FIR filter with coefficients confined to the ternary set: $\{-1,0,+1\}$. As the input to the ternary filter is in single-bit format, hardware implementation will be significantly efficient in the sense that each multiplication operation can be implemented with either a couple of logic gates or a simple lookup table [7]. The structure of the non-adaptive ternary filter is shown in Fig.(1). The ternary filter output $y(k)$ is given by the convolution of ternary taps $\{h(i)\}$ (or simply $\left\{h_{i}\right\}$ ) and the input signal $\{x(k)\}$ as follows:

$$
y(k)=\sum_{i=0}^{M} h_{i} x_{k-i}
$$

where $M$ is the order of the filter. The tap values are generated via $\Sigma \Delta$ modulation of a target impulse response. The digital $\Sigma \Delta \mathrm{M}$ used for this purpose must have a tri-level output, and must have a flat signal frequency response over the bandwidth of interest [6]. There are two other algorithms to generate the non-adaptive ternary tap coefficients from a specific target impulse response, such as dynamic programming and mini-max techniques [4].

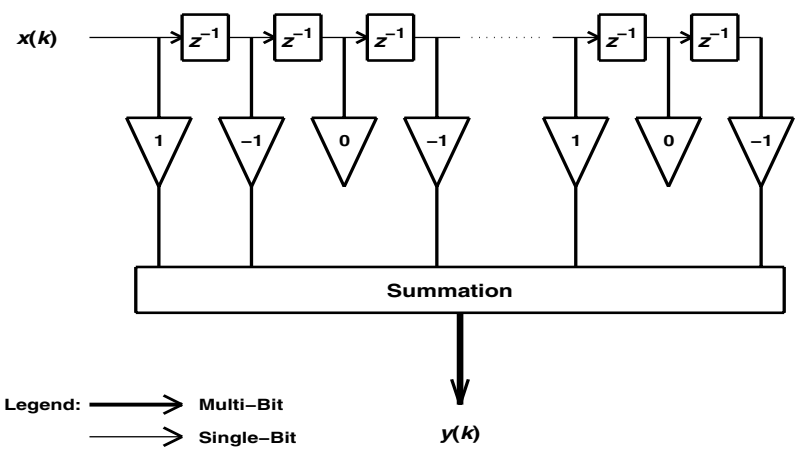

Fig. 1. Structure of the ternary filter.

\section{An AdAPTIVE Algorithm}

To the best of our knowledge, the issue of ternary adaptive algorithm has not been addressed yet, and is considered as an unresolved problem. We propose here, a new structure inspired from the well-known LMS adaptive techniques. Fig.(2) illustrates the ternary structure that carries out the proposed adaptive algorithm. We assume that the received (observed) signal, $r(n)$, is in single-bit format that represents the digitized original signal $x(t)$ (assumed in this paper to be a sinusoid) distorted by white Gaussian noise $w(t) \in \mathcal{N}\left(\sigma^{2}, 0\right)$. The same scenario in Fig.(2) can be used to represent a baseband version of a digital single-bit communication system with bandpass modulation [10].

The symbol $\hat{x}$ stands for the multibit estimated signal, and $y(i)$ is the estimated signal in single-bit format. The operation of this adaptive structure can be described as follows. The ternary system is comprised of $M$ adaptable taps and operates at an oversampling rate $R(R=64,128, \ldots)$. This requirement has already been met as the input signal is assumed to be $\Sigma \Delta$ modulated. The single-bit estimated signal $y(i)$ is shifted into a shift register of 
length $M$. At any particular time instant $n$, the regressor vector $\mathbf{y}(n)$ can be expressed as:

$$
\mathbf{y}(n)=\left[y_{M-n \Delta-1}, \ldots, y_{n \Delta}\right]
$$

where $\Delta$ is a time delay which denotes the filter coefficients updating rate. Likewise, the regressor vector of the received singlebit signal can be retrieved directly from the delay line of the ternary filter and can be given as: $\mathbf{r}(n)=\left[r_{M-n \Delta-1}, \ldots, r_{n \Delta}\right]$.

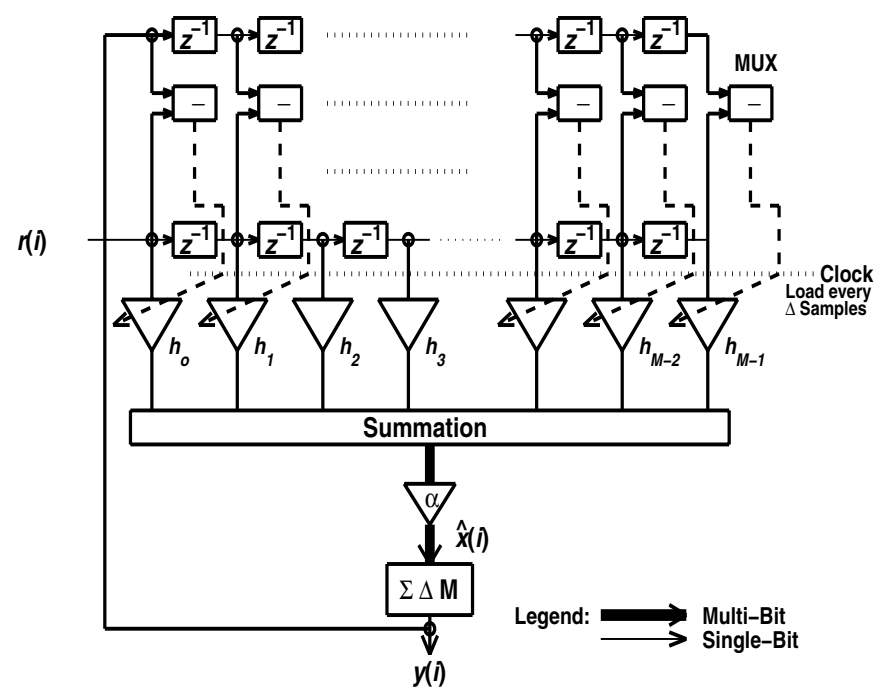

Fig. 2. Structure of the ternary filter.

This structure updates the ternary coefficients $\left\{h_{i} \mid i=\right.$ $0,1,2 . ., M-1\}$ once every $\Delta$ samples, where $\Delta$ is dependent on the oversampling ratio (here $\Delta=4 R$ ). This updating can be expressed as follows:

$$
\mathbf{h}_{n}=\frac{1}{2}\left(\mathbf{x}_{n}-\mathbf{y}_{n}\right) .
$$

The multi-bit estimated signal $\hat{x}$ is given as follows:

$$
\hat{x}=\alpha \mathbf{h}_{n-1} \mathbf{x}_{n-1}
$$

where $\alpha$ is a small positive parameter.

As $\hat{x}$ is in multi-bit format, a second-order standard $\Sigma \Delta$ modulator is used as shown in Fig.(3) to convert $\hat{x}$ to a bit-stream (re-modulation). This $\Sigma \Delta$ stage should have a flat frequency response in the band of interest such that the information in $\hat{x}$ is maintained. However, this stage will inevitably introduce noise to the output $y_{i}$ due to the quantization error $Q_{i}$. In addition, to preserve stability of the system, the value of $\hat{x}$ should be maintained within the dynamic range of the $\Sigma \Delta$ modulator. This can be guaranteed by introducing the gain parameter $\alpha$, which is a small positive value. The parameter $\alpha$ is related to the oversampling ratio $(R)$. This is so because the number of taps is proportional to $R$ [5]. For simplicity of implementation, $\alpha$ takes on negative powers of 2. It is found that optimum value for $\alpha$ is $\alpha=1 / 4 R$.

The instantaneous single-bit estimated output $y_{i}$ is given as follows:

$$
y_{i}= \begin{cases}+1 & \text { for } u_{i} \geq 0 \\ -1 & \text { for } u_{i}<0\end{cases}
$$

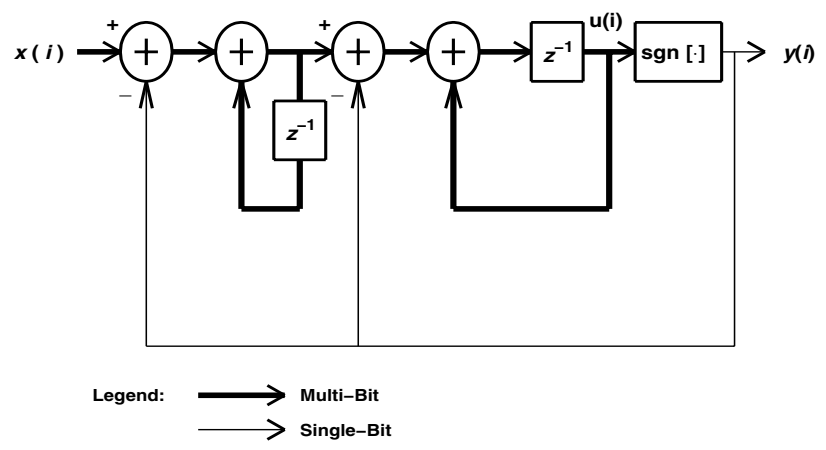

Fig. 3. Structure of the ternary filter.

where $u_{i}$ is the quantizer input of the $\Sigma \Delta$ stage and is given as: $u_{i}=u_{i-1}-y_{i-1}+\hat{x}_{i-1}$, [i.e., $y_{i}=\operatorname{sgn}\left(u_{i}\right)$ ].

Therefore, from (3), the filter coefficients vector can be given as:

$$
\mathbf{h}_{n}=\frac{1}{2}\left[\mathbf{x}_{n}-\operatorname{sgn}\left(\mathbf{u}_{n}\right)\right]
$$

From (5), it is evident that the elements of $\mathbf{h}_{n} \in\{0,+1,-1\}$

Now, if we utilize the linear model of the second-order $\Sigma \Delta$ modulator, then at any time instant $i$, the single-bit estimation can be expressed as follows:

$$
y_{i}=\hat{x}_{i-1}+Q_{i}
$$

where $Q_{i}$ denotes the quantization noise introduced by the $\Sigma \Delta$ modulator stage. Hence, the updating formula for the adaptive filter taps will be as follows:

$$
\mathbf{h}_{n}=\frac{1}{2} \mathbf{x}_{n-1}\left[\mathbf{I}-\alpha \mathbf{h}_{n-1}\right]-\frac{1}{2} \mathbf{Q}_{n}
$$

where $\mathbf{I}$ is an $M \times 1$ identity vector.

This adaptive ternary structure is very efficient from the hardware implementation point of view, as the ternary taps can be realized by using simple multiplexers. Moreover, the updating rate $\Delta$ can be achieved through the use of a conventional counter.

\section{Simulation And Discussion}

We have utilized MATLAB to simulate the performance of the adaptive structure shown in Fig.(2). Fig.(4) shows the the tracking performance of the adaptive ternary filter under the following conditions. The oversampling ratio is chosen as $R=128$, and the number of ternary coefficients is $M=2560$. The observed signal (input) $r_{i}$ is assumed to be the signle-bit digitized version of the original sinusoid $x(t)$ which is distorted by additive white Gaussian noise $w(t)$ with signal-to-noise ratio (SNR) of -8 $\mathrm{dB}$. The sinusoid has an amplitude $A=.5$ and a frequency $f_{o}=2000$ Hz. In Fig.(4), a $6^{\text {th }}$ order digital Butterworth filter is used to extract the single-bit estimated output. However, for accurate comparison, we didn't use the time domain to calculate the output SNR. Fig.(5) shows the corresponding spectra of both the received and estimated signals.

Now, to assess the performance of our adaptive ternary structure, we attempt to compare it with that of a traditional LMS Weiner adaptive algorithm under similar circumstances. We assume a Weiner filter with $N=R / M=20$ coefficients, operating on the same input signal. To be in the safe side, we assume the Weiner filter sampling rate as $4 \times$ Nyquest rate with infinite 


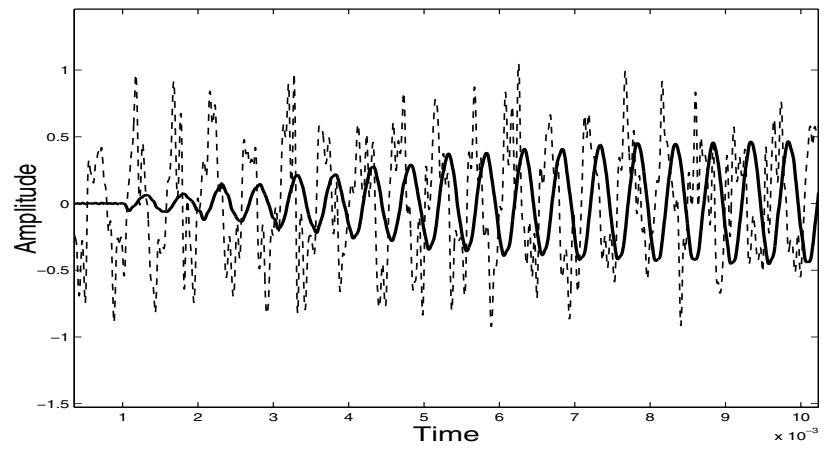

Fig. 4. The tracking response of the adaptive ternary filter. (solid):estimated output, (dashed):received input.

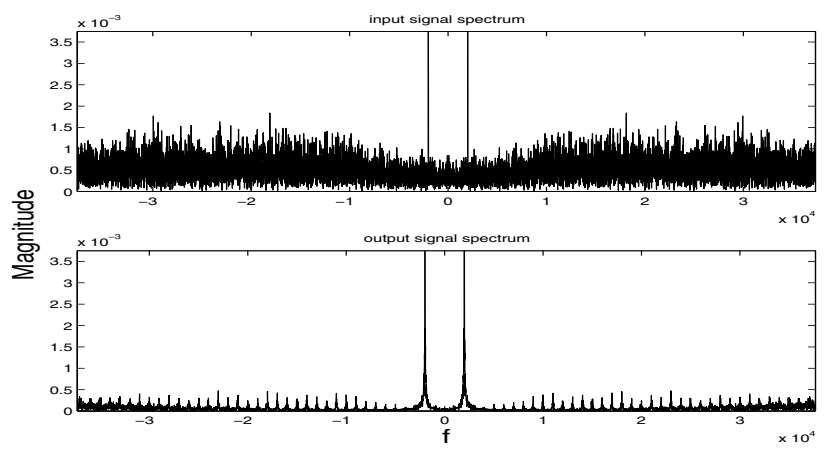

Fig. 5. The frequency spectra of the received signal (upper), and the estimated signal (lower).

bit resolution. Fig.(6) shows the the optimal value of the stepsize $\mu=0.0003$ in this case (i.e., $\mu$ that produces minimum meansquare error). The optimum $\mu$ is then used in the comparison.

In this comparison between the ternary and the LMS adaptive algorithms, we take the improvement (difference) in the output signal-to-noise ratio $\left(S N R_{o}\right)$ from the input $\left(S N R_{i}\right)$ as a performance measure. To ensure the proper accuracy, 51200 points have been used for this purpose. The sampling frequency in the ternary filter is $512 \mathrm{kHz}$, hence the frequency bin will be $10 \mathrm{~Hz}$. After extensive simulations, it is found that in ternary adaptive filtering, there is an average improvement in SNR of about 12 $\mathrm{dB}$, while in the case of traditional Weiner algorithm the average improvement in SNR was about $10 \mathrm{~dB}$. It was evident that the adaptive ternary filter is comparable in noise reduction to that of LMS Weiner filter.

\section{Conclusions}

In this paper we introduced an approach to solve the problem of adaptive ternary filtering. Despite the simple structure, simulation results showed that the proposed algorithm is parallel in performance to the multi-bit Weiner LMS algorithm. we expect that this approach will open the door for a wide range of applications for ternary systems.

\section{ACKNOWLEDGEMENT}

This work is supported by the Australian Research Council under the ARC Discovery Grant DP0557429.

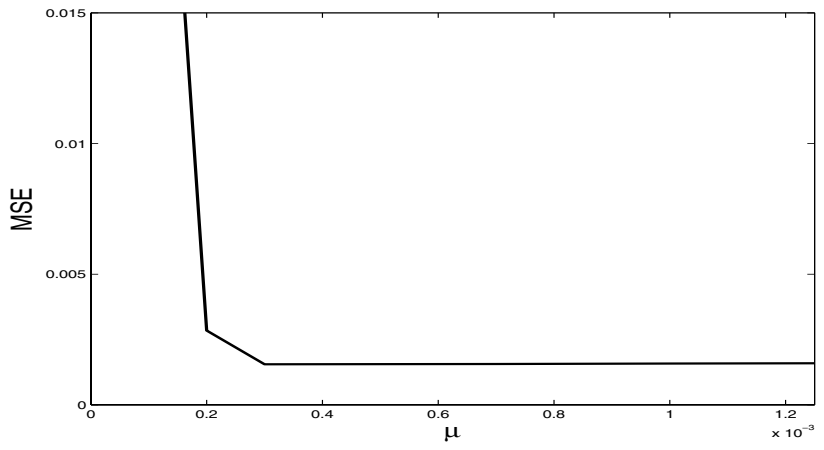

Fig. 6. The step-size $\mu$ versus the mean-square error of the LMS Weiner algorithm.

\section{REFERENCES}

[1] N. Benvenuto, L. E. Franks, and F. S. Hill, JR., "Realization of finite impulse response filters using coefficients $+1,0,-1$, IEEE Transactions on Communications, vol. COM-33, no. 10, pp. 1117-1125, Oct. 1985.

[2] P. W. Wong, "Fully sigma-delta modulation encoded FIR filters," IEEE Transactions on Signal Processing, vol. 40, no. 6, Jun. 1992.

[3] C. Dick, and F. Harris, "FPGA signal processing using sigma-delta modulation,” IEEE Signal Processing Magazine, pp. 20-35, Jan. 2000.

[4] B. R. Steele, Efficient Signal Processing Through the Use of SigmaDelta Modulation and Ternary Filters, PhD dissertation, RMIT University, Melbourne, 2003.

[5] A. C. Thompson, P. O'Shea, Z. M. Hussain, and B. R. steele, "Efficient single-bit ternary digital filtering using sigma-delta modulator," IEEE Signal Processing Letters, vol. 11, no. 2, pp. 162-166, Feb. 2004.

[6] A. C. Thompson, Techniques in Single-Bit Digital Filtering, PhD dissertation, RMIT University, Melbourne, 2004.

[7] A. C. Thompson, Z. M. Hussain, and P. O'Shea, "A Single-bit narrowband Bandpass digital filter," Australian Journal of Electrical and Electrnics Engineering, in press, 2005.

[8] A. Z. Sadik, Z. M. Hussain, and P. O'Shea, "Structures for Single-Bit Digital Comb Filtering," Proceeedings of the 2005 Asian Pacific Conference on Communications (APCC 2005), Perth, Australia, Oct. 2005.

[9] A. Z. Sadik, Z. M. Hussain, and P. O'Shea, "A Single-Bit Digital DC-Blocker Using Ternary Filtering," Accepted for IEEE TENCON 2005, Melbourne, Australia, Nov. 2005.

[10] J. G. Proakis et al, Contemporary Communication Systems Using Matlab, 2nd Edition, Brooks Cole : Pacific Grove, 2003. 